\title{
Beware Of the Consequences of Continuous and Comprehensive Evaluation Implications for Students' Learning
}

\author{
Garima Bansal \\ (Asst. Prof., Miranda House, University of Delhi; Ph.D Scholar, CIE, University of Delhi)
}

\begin{abstract}
Continuous and Comprehensive Evaluation (CCE) is conceptualized as a way of reforming the traditional examination regime, particularly board examinations, in India which has gained quite notoriety owing to its prime focus on education selection and certification. It is argued in this paper that though CCE is perceived as a panacea by making provisions of pupil assessment on a regular and comprehensive basis but in an examination driven culture its' vision does not translate into reality owing to several reasons. This paper delineates the impact of CCE on students' learning by utilizing an interpretative research methodology and offers suggestions to improve the assessment scenario for larger good.
\end{abstract}

\section{Background}

High stakes examinations have often been criticized for their negative impact on pupils' learning, specifically, promoting rote memorization of the content which often fails our students in real world problem solving situations. Position Paper on Examination Reforms (NCERT, 2006) notes that large software companies (NASSCOM) in India point out " 19 out of 20 graduate applicants and 6 out of 7 post-graduate applicants are unemployable. They simply lack requisite problem solving skills..." (p.5) indicating that our education system somewhere lacks in creating minds who can think creatively, divergently, and analytically. This concern with inadequacy of education system to instill new world skills (Broadfoot, 2008) are not confined to India only but shared by the entire world. Harlen (2008) while citing OECD (1999) pointed out that, 'students cannot learn in school everything they will need to know in adult life. What they must acquire is the prerequisites for successful learning in future life' (p.9). Learning how to learn can only be achieved if effective learning practices are incorporated in students' curricular experiences (Black et al., 2006).

However, only reforming curriculum and pedagogical practices without changing ways of assessment associated with it is bound to create ripples of tension in the education system. These ripples can only be pacified by a parallel reform in assessment. In addition, assessment is often found to exert a controlling influence on curricular and pedagogic practices by promoting 'teaching to the test'. In wake of this realization, several policy documents since long have been advocating to incorporate assessment reforms and move towards the introduction of a comprehensive scheme of pupil evaluation that is child friendly, classroom centric and assesses processes of learning as they unfold on a regular basis. To support my point, the report of Secondary Education Commission (1954) urged that 'the emphasis on all- important annual examination should be reduced' and school records should be maintained to give a 'true picture of learners' all round progress throughout the year by reducing the weightage of external examinations and numerical marking. Similarly, Kothari Commission (1964) pushed for assessment reform at all stages and CABE Committee on Examination Reform (1970) made wide ranging recommendations in this regard. In the similar wake, National Policy of Education (1986) advocated comprehensive methods of assessment that assess students on a continual basis rather than it being a secluded year end affair. This report also considered examination as a means of improving quality of education. It was followed by Programme of Action (POA) recommending several short term and long term measures for carrying out examination reform at school level. Furthermore, $19^{\text {th }}$ Annual Conference of the Council of Boards of School Education in India (1990) recommended the need of continuous and comprehensive internal evaluation and placing assessment in the classrooms in the hands of teachers. In continuation, NCF (2005) observed that "In the medium term, we need to be able to increasingly shift towards school-based assessment, and devise ways in which to make such internal assessment more credible. Each school should evolve a flexible and implementable scheme of Continuous and Comprehensive Evaluation (CCE), primarily for diagnosis, remediation and enhancing of learning. The scheme should take, into account the social environment and the facilities available in the school" (p. ).

These viewpoints gathered momentum, post the advocacy of NCF 2005, and CBSE in its affiliated schools launched the Continuous and Comprehensive Evaluation (CCE, henceforth) model of pupil evaluation. In addition, various state boards of school education also have been implementing curricular and examination reforms based on the recommendations of NCF 2005. This model of assessment has the characteristic feature of formative assessment (FA, henceforth) interwoven in it. It mandates teachers to carry out formative assessment 
of pupils on an ongoing basis which involves:

- $\quad$ Establishment of a classroom culture that encourages interaction and the use of varied assessment tools;

- $\quad$ Establishment of learning goals, tracking of an individual student progress;

- $\quad$ Use of varied instruction methods to meet diverse students' needs;

- $\quad$ Use of varied approaches to assessing student understanding;

- $\quad$ Feedback on student performance and adaptation of instruction to meet identified needs;

- $\quad$ Active involvement of students in the learning progress. (OECD, 2005)

All these features, undoubtedly, seem to make the process of assessment learner friendly by eradicating the stress associated with it, a move in the direction of 'Learning without Burden' () and shifting the focus of assessment from 'passing the examination' to 'learning for its own sake' (Dore, 1997).

However, assessment being a social practice (Broadfoot, 1996) derives its implications from the context in which it is practiced. Policy borrowed from elsewhere, even though it may be student centric, may not see any light in the Indian context as social, cultural, political and economic factors are different which transpires while interpretation and implementation of the examination reform. This paper tends to recognize 'What has been intended for in terms of pupils' learning?' through CCE and 'What has been the outcome?'. Limitations of assessment reform while being translated into practice are identified and possibilities are discussed vis-à-vis students' learning.

\section{Transforming CCE vision into practice: The Reality}

To bring any educational reform to fruition is not a runaway victory. It requires deeper investments to be made in the various social, political, and economic spheres so that all may considerably contribute to transform the vision into reality. In school context, specifically, focusing upon the secondary education where CCE was initially launched, collaboration among various competing stakeholders seems to be inevitable for the successful implementation of CCE so that it can be placed as a cornerstone for the pursuit of lifelong learning.

Exploring the De Facto position of CCE in schools:

CCE was instated by CBSE in its affiliated schools in the mid session in the month of October, 2009.

Though there had been plans of inception of such an assessment reform for long but when will it become a policy concern was never sure among the school teachers and administrators. Due to the rush with which CCE became a mandate, teachers and all other stakeholders actually got awestruck as in the absence of adequate training and practice, implementing a reform seemed nearly impossible. Consequently, CCE witnessed variable interpretations of the policy mandates and started being practiced differently across different school contexts, ranging from convergent to divergent forms of FA (Torrance, 1998). This misconstrued framework, as found in the field relating to convergent FA, has become the buzzword in the educational discourses in India and in school settings particularly. The focus of this study is to unpack the impact of this form of CCE interpretation on pupils' learning.

\section{Evolving a Theoretical Framework:}

The major intent of the any educational act is to carry out assessment for the sake of increasing pupils' learning. This framework of assessment, as opposed to measurement approach to assessment, aims to embed assessment within the teaching- learning processes. A compact yet comprehensive list of the assessment processes is provided by Assessment for Learning: 10 Principles that are summarized as under:

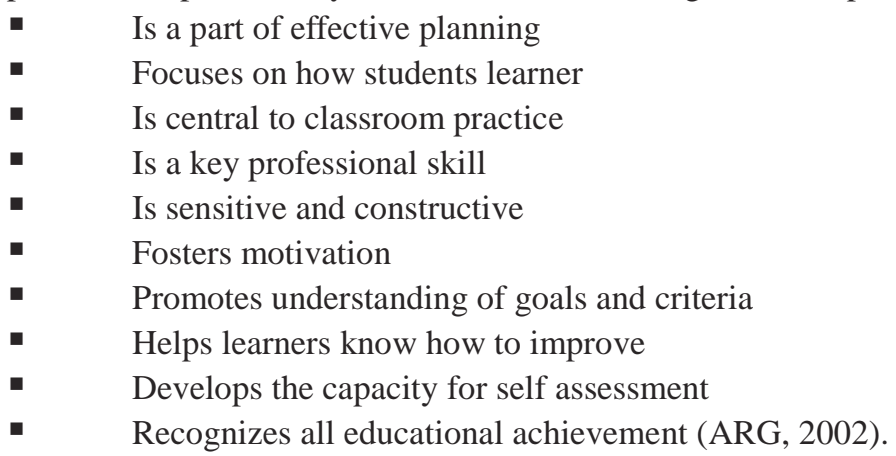

As clear that the focus of assessment is necessarily the pupil 'learning' which involves in- depth conceptual understanding, ability to extend learning that had taken place in school to the real world situation and not just the ability to regurgitate certain facts and fixed procedures on answer sheet only to be forgotten as soon 
as the testing ends. Research has reported the following aspects of pupils' learning that are hoped to be developed through utilizing classroom based assessment practices:

- $\quad$ Assessment will increase pupils accountability towards themselves:

Huge amount of research echoes that carefully planned classroom assessment develops skills of self awareness, and self regulation among learners. They develop 'strategic awareness' (Broadfoot, 2008), that is, learners are empowered to articulate thoughts' about one's own learning and gain a sense of ownership while engaged in assessment. In addition, classroom based assessment transfers the locus of control of learning and assessment to the pupil themselves (Black \& William, 2003) which not only supports learning in cognitive domain but also in affective dimensions, such as, self esteem, self efficacy, goal orientation etc..

- $\quad$ Pupils perceiving assessment as an enjoyable process:

Research reports that alternative assessment characterized by 'authentic' tasks (such as portfolios, self assessment, peer assessment, projects and other non-examination assessment ) are positively evaluated by students thus making learning more powerful and realistic (Brown \& Hirschfeld, 2008). While citing Slater (1996), they further added that students remarked that the material learned for portfolios is learned for longer period of time as during the prolonged process of assessment the data gets internalized. It has added benefits of achieving 'learnability' which is associated with an enthusiastic, critical and curious approach towards learning as opposed to 'stuckness' where learner is a passive and uninterested participant (Broadfoot, 2008).

- $\quad$ Assessment as a co-operative act and downplaying competition

Black (1998b) reported that formative measures of assessment have significantly helped the (so-called) low attainers more than the rest, thereby reducing the spread of attainment while also raising it overall. This illustrates that formative assessment necessarily works for uplifting the educational quality and involves learners in the pursuit of lifelong learning. It occurs by the way of creation of a classroom climate of mutual respect and fairness (Osborm et al. 2003 as cited by Broadfoot, 2008). Such an environment encourages interactive and collaborative learning and the role of teacher is to 'engineer' such co-operative learning opportunities for their pupils.

- $\quad$ Using Feedback to support learning:

The essence of classroom assessment lies in providing relevant and quality feedback to enable learners to 'close the gap' between their current position and desired achievement. Feedback needs to be process oriented as opposed to focusing upon products. The focus of the assessment has to shift away only from the 'matured' functions to those that are still in the process of 'maturation' (Vygotsky, 1978) and can be ripened in a scaffolded and assisted learning environment. Furthermore, assessment conversations, which embed assessment into the teaching- learning landscape, are crucial to provide quality feedback. And it needs to be ensured that for formative assessment to be complete, feedback information has to be used (Black \& William, 1998).

These key points illustrate and provide a guiding framework through which impact of CCE on pupils' learning is explored. It is hoped that by the means of examination reform pupils will feel intrinsically motivated to carry out learning in a non-threatening environment throughout their lives.

\section{Method}

The research used an interpretive research methodology (Erickson, 1998), and involved 9-10 teachers of science and one of their classes in each case, a total of ten classes. In the first phase of the project, the ten teachers and thirty-one of their pupils (at least three from each class) were interviewed to ascertain their views on CCE and its consequent implications on pupils' learning practices, patterns etc. Specifically, information was sought from the pupils by asking their views about assessment reform, how it has changed the assessment landscape as compared to their earlier classes, what is the role of their teachers in the assessment process and how did their teachers help them with their learning now, and what is their role in assessment when CCE has come into place?

The second phase of the project involved an in-depth exploration into the nature of the classroom assessment practices that supported learning. Each class was observed throughout a science unit, a time of three to five weeks. Post classroom observations, pupils were interviewed individually or as a group, depending on the feasibility requirements.

Pupils' comment had a key feature of comparing traditional examination practices with FA approaches. Though most of the times they seemed confused and made more general (usually negative) statements about teacher assessment practices initiated by CCE approaches yet few of them placed CCE in learning orientation towards assessment.

In this article, observational and pupil interview data have been combined to illustrate the ways pupils perceived their engagement in 'assessment for learning' and how CCE has impacted pupils' learning. The trustworthiness of the data and data interpretation presented here was ensured through prolonged engagement in 
the classroom (Cowie, 2005).

\section{Findings}

The purpose behind any education reform is to increase pupils' learning and ensure by adopting appropriate measures that pupils are at least learning what was intended for (Klenowski, 2006). Furthermore, as the framework suggests that students being assessed through CCE will be assessed continually and may find assessment an enjoyable process as opposed to stress and anxiety associated with formal measures of examining students' achievement. The upcoming sections will illustrate the impact of CCE on pupils' learning:

- How Much Holistic Understanding is being developed?

Classroom based assessment is seen as a way to encourage holistic conceptual understanding. It aims at placing the learning at centre stage of all the educational practices. Apart from the conceptual understanding, it focuses on developing a repertoire of 'learning to learn' (OECD, 2005) skills of pupils. This conceptualization, however, has been translated differently in practice. Field suggests that CCE is being interpreted as a massive increase in the quantity of short atomized tests which has encouraged 'reductionist approach to knowledge acquisition'.

Ss: Ma'am, kitne FA ke liye padhein....Ek din mein chaar-chaar FA hot hein...

Performing well on each FA that will eventually pool into the CGPA at the end of term is the new educational goal. It appears that in a rush to succeed on successive FAs, an evolution towards the conceptual whole seems to be missing leading to further 'atomization of learning' (reinforcing one of the prime critiques of formal testing). It further creates extrinsic motivation towards task as behavioristic principles of punishmentreward orientation towards FAs are in place.

- Is assessment integrated in classroom teaching- learning?

Shephard (1996) argued that assessment and teaching should be so interwoven in a class practicing FA that it becomes difficult to suggest where one ends and the other begins. Also, the inherent belief, while planting assessment in the classroom framework, is that it will eradicate the stress associated with testing situations and provide teachers' timely assessment evidence to provide feedback on. This feedback would help the learners there and then instead of postponing it to some other point of time.

However, in practice, the separation between teaching and assessment contexts pertains. Students pointed out that FA always occurred at the end of a chapter, a practical, a concept; or a term etc. that is 'at the end' ruled the scene. Each and every curricular portion was full stopped with a FA. That is instead of intermingling FA in the teaching- learning process, it is relegated towards the end of the session- this conception misses the essence of FA which urges to focus on teachable moments, evolving and deploying assessment conversations for diagnosing learners' needs instead of using assessment to label the children based on their outcomes.

- Towards creating a 'stress free' culture of evaluation....

It was observed that teachers, in a bid to embed assessment in their classrooms, took their CCE Record Books along with them and students' each and every move was noted in it. It was observed during classroom observations that students' responses to teachers' question were consistently being marked in the Record Books. It amounted to a constant pressure on the teachers to constantly focus upon who is doing or saying what and on students as they have to be correct/ at their best since they were being marked.

In addition, comprehensiveness is being interpreted as a constant watch or in Foculadeian terms 'surveillance' (Gipps, 1996) on all the dimensions of pupils learning. Pupils pointed out that

It seems I'm watched by an external eye, may it be in class, during recess, or in playground.....hmnnn..... it is stifling at times.

Pupils pointed out that their behavior gets contrived as soon as they realize that an external eyereferring to their teacher- was watching them. Infact, it is not only the case with children but common to all the human enterprise that out actions get artificially crafted as soon as we realise that we are being watched. Similarly, learning becomes a stressful affair when a teacher (who is interpreted as having a dictatorial role) constantly watches them.

- Widening the gulf between 'good' and 'poor' students:

Teachers as well as students pointed out that owing to extreme stress of FAs, few students find themselves unable to deal with the pressure may be due to sheer inability to prepare for, say 5 , tests in a day. Therefore, students have developed an avoidance towards the academic work perceiving it to be irrelevant. Brown \& Hirschfeld (2008) noted that students may perceive assessment as irrelevant if it is thought as being bad or unfair. They reported on research elsewhere (Duffield and Spencer, 2002) arguing that perceived subjectivity and lack of pofessionalism in assessment has led tertiary students to view assessment as arbitrary, irrelevant, inaccurate and simply a necessary process for accruing marks. This group of students develops a casual attitude towards academic work and stops performing even on recurrent trials of FA. 
While on the other end of continuum exists so- called 'good' students who work extremely hard for each and every low stake FA so that the cumulative grade achieved by them is higher. Even this lot of students finds CCE demotivating as they say

Ma'am, kitna bhi padh lo, grade wahi rehta hai, pata nahin chalta kisne kitna better kiya

This statement is evocative of the fact that these pupils missed the extrinsic motivation derived from formal testing and probably CCE is not able to generate an intrinsic motivation in them.

These findings represent that CCE's conception has not being adequately translated into the desired ways of pupils' learning.

\section{Discussion}

In this paper, I have attempted to develop a critical understanding about the ways in which assessment reform, CCE, has impacted students' learning. there are always pros and cons of any educational initiative undertaken and all of them cannot be charted out at the outset. nevetheless, it is crucial to anticipate certain issues that may emerge and take a stock of things to ensure that practices are enroute. It is time to ask ourselves certain vexed questions ' Does CCE really improving the 'quality' of educational outcomes? is it making our young learners better equipped with new world skills? and Is the enormous amount of money,efforts, time, expertise acheiving what was intended for? or is CCE practices taking us in an unintended, unassumed direction which is very different: strangulating learners' crerativity, demoralising professionals by enhancing their burnout and eventually leading us as a society towards a dead end.

Therefore, while taking an account of practices that have come up with CCE it could be suggested that in a testing obsesssed school system it is difficult to realise the potential of Assessment for Learning (AfL) practices. Probably, the reason behind it seems to be extreme faith in assessment tools and technologies often associated with high stakes external testing systems. Furhter, the sitaution becomes intractable due to the dissonace between assessment technologies being used for summative vis-a-vis formative purposes.

This extreme faith in high stakes examination seems to have its origin in the capabiltiy of assessment outcomes to label (Filer, 2000) the pupils for life and ration future opportunities for them. In such a scenario, similar to countries like Nepal and Hong Kong, making the stakeholders realize the value of assessment for learning as compared to assessment for selection seems to be a difficult proposition ( the researcher when engaged in a Focused Group Discussion found learners asking about 'ma'am, iske number lagenge kya?' pointing that any activity is undertaken if and only if it has stakes associated with it). in such cases, even the most change committed democratic teacher gets demoralised and resort to normalisation of their professioanl activiites that is in keeping with the traditional definitions of what constitutes good practice and desirable learning outcomes often measured in terms of marks.

Also, parents do not seem to value the FA approach towards assessment and coax their children to study to the summative test. For instance, parents whose children are assessed through CCE point out that 'number aaye to kuch pata chale, grade pakda dete hain' which reflects the uneasiness and lack of acceptance of CCE model by the parental community. Eventually, studying patterns that may bring 'good result' in summative assessment is valued and other forms of pupils' task engagement are suppressed. this traps all the stakeholders in a self perpetauting vicious circle and it is hard to find any exit point out of it.

Furthermore, while pointing to pupils' learning as promoted through the examination reform, it came to the fore that instead of developing self regulated lifelong learners, CCE is creating a generation of pupils who study small packets of content to the test and forgets it as soon as the assessment is over. Pupils reported that in FAs we prepare power point presentations and simply do CCP (Cut, Copy and Paste). They confessed that they hardly read the material they pasted on the slides and simply read it aloud while presenting. This form of task engagement is reflective of the performance orientation towards assessment and pupils working with extrinsic motivation to pass the test.

However, there lies the possibility of realizing the 'real' change'- even if it seems unlikely- through CCE if further investments are made in teacher development, provision of adequate support to schools, incentives to innovation, collegial support and closer ties between research, school and policy are made. All the more in depth planning to deal creatively with logistical and organizational barriers is recommended. The prime focus of assessment has to be shifted from 'marks' to 'learning' and it can only happen if the higher education sector and job opportunities lay great stress on recruiting lifelong learners with the ability to critically analyse, provide divergent solutions and transfer the classroom learning to real life problems. 


\section{References}

[1]. Black, P. \&. (1998). Assessment and classroom learning. Assessment in education: princilples, policy and practice, 5(1).

[2]. Black, P. W. (2001). Inside the Balckbox: Raising standa0rds through classroom assessment. BERA final draft.

[3]. Broadfoot, P. (2008). An Introduction to Assessment. continum.

[4]. Brown, G. T. (2008). Students' conceptions of assessment: Links to outcomes. Assessment in Education: Principles, Policy and Practice, 15(1), 3-17.

[5]. Cowie, B. (2005). Pupils' commentary on assessment for learning. Curriculum Journal, 16(2), 137-151.

[6]. Dore, R. (1997). the argument of diploma disease. Assessment in Education: Principles, Policy and Practice, 4(1), 23-32.

[7]. (2005). Formative Assessment: Improving Learning in Secondary Classrooms. OECD.

[8]. Klenowski, V. (2006). Learning Oriented Assessment in the Asia- Pacific region. Assessment in Education: Principles, Policy and Practice, 13(2), 131-134.

[9]. NCERT (2006). Position Paper National Focus Group on Examination Reforms. New- Delhi: National Council of Educational Research and Training (NCERT).

[10]. Torrance, H. \&. (1998). Investigating Formative Assessment: Teaching, Learning and Assessment in the classroom. Buckingham: Open University Press. 